\title{
Mapeamento Sistemático sobre o uso de Visualização de Dados para Análise Processual da Aprendizagem em Ambientes Virtuais
}

\author{
Raphael A. Dourado ${ }^{1}$, Rodrigo Lins Rodrigues ${ }^{2}$, Nivan Ferreira ${ }^{1}$, Alex Sandro \\ Gomes $^{1}$ \\ ${ }^{1}$ Centro de Informática, Universidade Federal de Pernambuco (UFPE) \\ Av. Jornalista Aníbal Fernandes, s/n - Cidade Universitária - 50.740-560 - Recife - PE \\ ${ }^{2}$ Departamento de Educação, Universidade Federal Rural de Pernambuco (UFRPE) \\ Rua Dom Manoel de Medeiros, s/n, Dois Irmãos - 52171-900 - Recife - PE \\ rasd2@cin.ufpe.br, rlr@ded.ufrpe.br, \{nivan,asg\}@cin.ufpe.br
}

\begin{abstract}
This paper presents a systematic mapping on the use of data visualization techniques to analyze learning processes in virtual learning environments. The research questions adopted sought to identify the aspects of learning processes that have been the subject of research, the visualization techniques used, how the proposals have been empirically validated, and the learning theories that are being used to guide these studies. The results obtained with this study reveal that few works effectively prioritize the analysis of processes rather than the products of learning, propose sophisticated visualizations and use learning theories to ground their proposals.
\end{abstract}

Resumo. Este artigo apresenta um mapeamento sistemático sobre o uso de técnicas de visualização de dados para análise dos processos de aprendizagem em AVAs. As questões de pesquisa adotadas buscaram identificar que aspectos dos processos de aprendizagem têm sido alvo de investigação, as técnicas de visualização utilizadas para tal, a forma de validação empírica das propostas $e$ as teorias da aprendizagem que estão sendo utilizadas para guiar estes estudos. Os resultados obtidos com este mapeamento apontam para uma carência de trabalhos que efetivamente priorizem a análise dos processos ao invés dos produtos da aprendizagem, proponham visualizações sofisticadas $e$ utilizem teorias da aprendizagem para alicerçar suas propostas.

\section{Introdução}

A crescente disponibilidade de dados relativos à interação de alunos e instrutores em Ambientes Virtuais de Aprendizagem (AVAs) fomentou o surgimento de uma nova área de pesquisa, conhecida como Learning Analytics. Esta área se propõe a medir, organizar, analisar e reportar dados de estudantes e seus contextos de aprendizagem, com o objetivo de entender e otimizar a os processos de aprendizagem e os contextos onde eles ocorrem [Siemens \& Gasevic 2012].

Uma das formas mais efetivas de organizar e comunicar estes conjuntos de dados, de forma a torná-los úteis aos diversos atores envolvidos no processo de ensino-aprendizagem, é por meio de visualizações interativas, o que tem levado ao desenvolvimento de uma subárea de pesquisa dentro de Learning Analytics chamada de Visual Learning Analytics [Vieira et al. 2018]. Professores e gestores podem utilizar 
VII Congresso Brasileiro de Informática na Educação (CBIE 2018)

Anais do XXIX Simpósio Brasileiro de Informática na Educação (SBIE 2018)

visualizações como ferramenta para melhor compreender o desempenho dos estudantes, fornecer feedback apropriado e otimizar o processo educacional. Já os estudantes podem utilizá-las como instrumento para auxiliar na autogestão da aprendizagem.

No entanto, apesar de muito se falar em "análise dos processos de aprendizagem" na literatura de Learning Analytics, alguns pesquisadores acreditam que grande parte dos trabalhos desenvolvidos até o momento estão mais focados em analisar os produtos da aprendizagem que os processos de aprendizagem propriamente ditos [Vieira et al. 2018, Lockyer et al. 2013]. Esta supervalorização dos produtos em detrimento dos processos também tem sido criticada por pesquisadores da área de Psicologia Cognitiva, especialmente aqueles ligados à corrente sociohistórica fundada por Lev Vygotsky [Mello 2004]. Teóricos como Barbara Rogoff [Rogoff 2003], Jean Lave e Etienne Wenger [Lave \& Wenger 1991] apontam em suas teorias a característica intrinsecamente processual da aprendizagem e suas relações com a mediação social.

Desse modo, percebe-se que visualizar os processos de aprendizagem não tem sido uma tarefa fácil para usuários de ambientes virtuais de aprendizagem. Para melhor entender tal dificuldade, o presente estudo visa verificar, através de um mapeamento sistemático, como os trabalhos da área de Visual Learning Analytics têm abordado a questão da análise processual da aprendizagem. As próximas seções deste artigo estão organizadas da seguinte forma: a Seção 2 discute conceitos relacionados à análise processual da aprendizagem em AVAs através de técnicas de visualização de dados; a Seção 3 apresenta o método utilizado para conduzir o mapeamento sistemático; a Seção 4 apresenta e discute os resultados; e por fim a Seção 5 resume o estudo e aponta as lacunas identificadas na literatura e oportunidades para trabalhos futuros.

\section{Análise processual da aprendizagem em AVAs com Visual Learning Analytics}

Segundo Vieira et al. (2018), a maioria dos estudos na área de Visual Learning Analytics são do tipo "checkpoint analytics". Este termo, cunhado por Lockyer et al. (2013), representa análises que são mais focadas em dados "fotográficos", ou seja, quantificadores que supostamente revelam se um estudante cumpriu os pré-requisitos para alcançar os objetivos de aprendizagem: métricas de acessos a materiais e ao ambiente, envio de tarefas, postagens em fóruns, entre outros. Segundo os autores, embora possam ser importantes indícios de engajamento, estes dados não são suficientes para compreender os processos de aprendizagem dos estudantes.

O contraponto ao "checkpoint analytics" seria o que Lockyer et al. (2013) chamam de "process analytics". Este tipo de análise busca investigar as etapas percorridas pelos estudantes para completar uma determinada tarefa. Lockyer cita como exemplos de técnicas que podem ser utilizadas para este fim a análise de redes sociais e a análise de conteúdo, porém uma área emergente de pesquisa que também pode servir a este fim é a Mineração de Processos Educacionais.

A Mineração de Processos Educacionais (ou Educational Process Mining EPM) [Bogarín 2018] é uma subárea da Mineração de Dados que busca extrair conhecimentos relacionados aos processos educacionais a partir de conjuntos de dados, em geral aqueles armazenados por plataformas virtuais. Dentre as diversas técnicas 
VII Congresso Brasileiro de Informática na Educação (CBIE 2018)

Anais do XXIX Simpósio Brasileiro de Informática na Educação (SBIE 2018)

utilizadas em EPM, está a visualização de dados, que pode ser utilizada tanto como forma de representar visualmente os padrões identificados por algoritmos de aprendizagem de máquina, quanto como ferramenta em si mesma para descoberta de padrões - especialmente através de recursos de interatividade, que permitem ao usuário explorar os conjuntos de dados e construir, validar ou refutar suas próprias hipóteses.

De forma a identificar as lacunas de pesquisa relacionadas a visualização de processos de aprendizagem em AVAs, foi definido um protocolo para mapeamento sistemático da literatura, o qual é descrito em detalhes na próxima seção.

\section{Método}

Um mapeamento sistemático da literatura, segundo Arksey \& O’Malley (2005) tem por objetivo fornecer uma visão ampla de determinada área de pesquisa, a partir da quantificação, categorização e análise dos estudos publicados. A primeira etapa deste processo consiste em definir o protocolo que irá guiar a pesquisa bibliográfica. Esta seção descreve o protocolo utilizado no presente estudo, o qual segue a metodologia proposta por Petersen (2007).

\subsection{Questões de Pesquisa}

A questão central que este estudo busca responder pode ser enunciada como "Que abordagens, técnicas, e suportes teóricos têm sido utilizadas na literatura para conceber visualizações de processos de aprendizagem em AVAs?’. A partir desta questão central, foram definidas as seguintes questões específicas de pesquisa:

- Q1: Que aspectos dos processos de aprendizagem as pesquisas estão buscando analisar a partir dos dados armazenados nas plataformas de aprendizagem?

- Q2: Que técnicas de visualização têm sido utilizadas na concepção dos artefatos?

- Q3: As visualizações propostas foram submetidas a avaliação de atores envolvidos no processo educacional (alunos, professores, gestores) ou incorporadas à suas práticas?

- Q4: Que teorias da aprendizagem têm sido usadas para guiar a investigação?

A partir destas perguntas, foi definida a estratégia de busca para obtenção da lista de estudos primários. A próxima subseção descreve a estratégia adotada.

\subsection{Estratégia de Busca}

Foi utilizada a base Elsevier Scopus como fonte para a obtenção dos estudos primários. Esta escolha se justifica pelo fato deste repositório indexar grande parte da produção científica na área de pesquisa deste estudo, incluindo a IEEE Digital Library, ACM Digital Library, ScienceDirect, SpringerLink, dentre muitos outros. Os resultados da busca comprovaram que até mesmo trabalhos publicados em conferência regionais são indexados pelo Scopus.

A partir das palavras-chave da questão central e questões específicas desta pesquisa, combinados com termos correlatos e sinônimos, foi definido o critério de busca mostrado abaixo, escrito segundo sintaxe do motor de busca do Scopus: 
VII Congresso Brasileiro de Informática na Educação (CBIE 2018)

Anais do XXIX Simpósio Brasileiro de Informática na Educação (SBIE 2018)

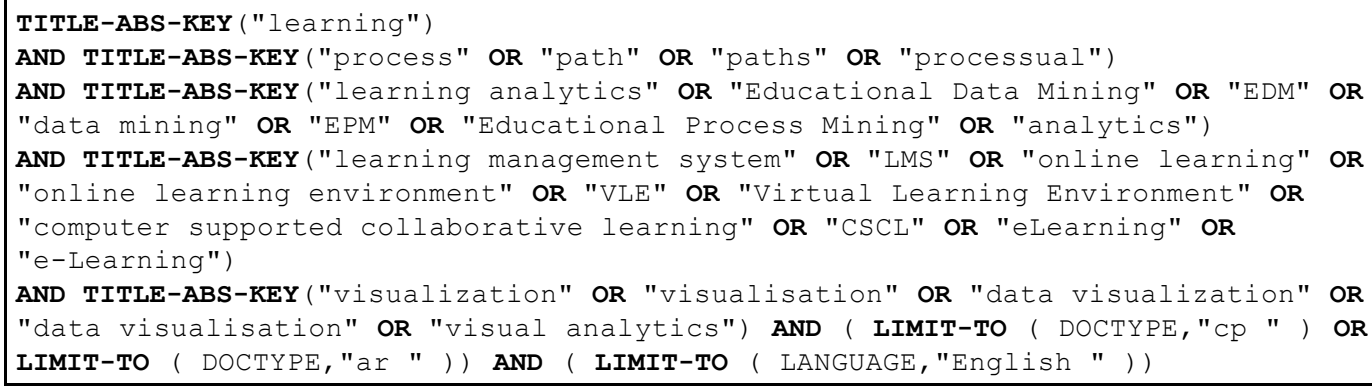

A partir deste critério de busca, foram retornados 71 trabalhos (51 artigos de conferência e 20 de periódicos). Quanto aos veículos de publicação, os mais frequentes neste conjunto foram o Springer Lecture Notes in Computer Science (7 trabalhos), ACM International Conference Proceedings Series (6 trabalhos) e o CEUR Workshop Proceedings (5 trabalhos). A distribuição dos trabalhos ao longo dos anos é mostrada na Figura 1, compreendendo o período entre a publicação do trabalho mais antigo e a data de realização deste estudo (julho de 2018). É possível observar um crescimento no número de publicações a partir do ano de 2012, com pico em 2015 e uma pequena queda a partir de então.

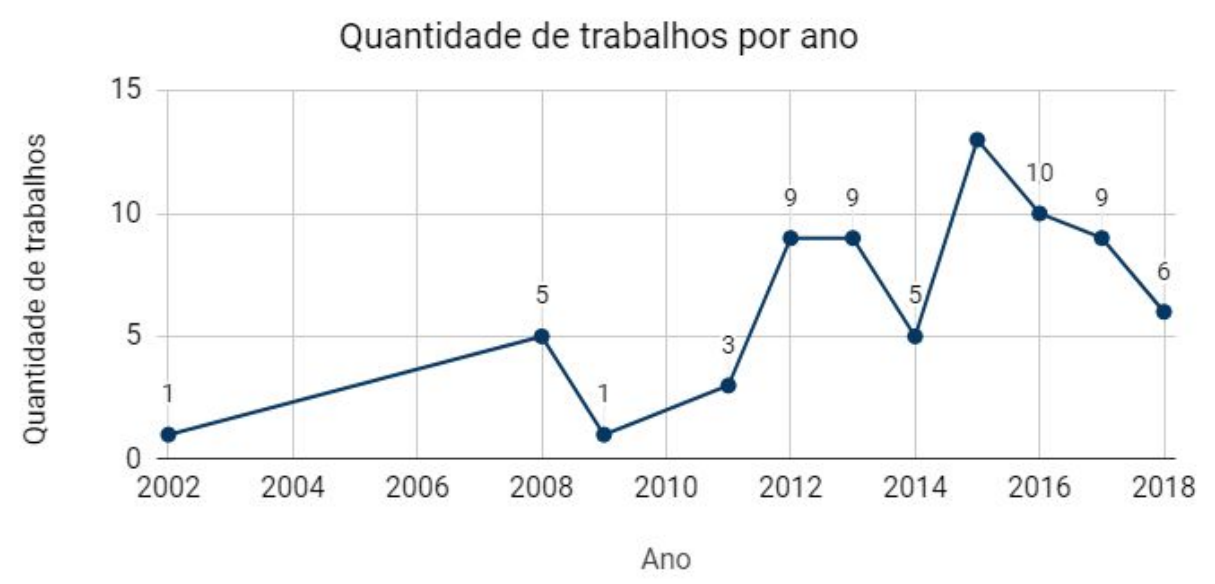

Figura 1. Distribuição, por ano, dos trabalhos retornados pelo critério de busca

Seguindo a metodologia proposta por Petersen (2007), após obtenção do conjunto inicial de trabalhos, deve-se definir e aplicar critérios de exclusão, de forma a remover os estudos que, apesar de conter as palavras-chave desejadas, não se enquadrem no escopo proposto no mapeamento. Dessa forma, a próxima subseção descreve os critérios exclusão adotados neste estudo.

\subsection{Critérios de Exclusão}

Com base nos objetivos deste estudo, foram adotados quatro critérios de exclusão para a seleção dos trabalhos retornados pela pesquisa na base Scopus:

- CE1 - O artigo cita genericamente "processos de aprendizagem" mas não coleta características comportamentais do processos de aprendizagem dos alunos, nem propõe uma ferramenta/método de análise voltada para este aspecto; 
VII Congresso Brasileiro de Informática na Educação (CBIE 2018)

Anais do XXIX Simpósio Brasileiro de Informática na Educação (SBIE 2018)

- CE2 - A visualização surge no artigo apenas como forma de comunicar os resultados experimentais da pesquisa, e não como uma proposta de artefato que seja utilizado para identificar características dos processos de aprendizagem;

- CE3 - O artigo não está inserido no domínio "educação", ou trata de treinamento corporativo;

- CE4 - É um artigo resumido, tutorial técnico, ou escrito em outro idioma que não o Inglês ${ }^{1}$.

Uma vez definidos estes critérios de exclusão, foi realizada a leitura do título, resumo e palavras-chave de cada artigo (e em alguns casos, onde estes itens não eram suficientes para aplicação dos critérios, foi lido também a introdução e conclusão) e excluídos os artigos que satisfizesse pelo menos um dos critérios. O critério CE4 se fez necessário pois, mesmo sendo solicitado ao motor de busca do Scopus que excluísse artigos resumidos ou não escritos em Inglês, alguns trabalhos desta natureza ainda foram retornados.

Após esta etapa de filtragem, restaram apenas onze estudos [Gelan et al.. 2018, Zhang et al. 2018, Hernández-García et al. 2015, Gómez-Aguilar et al. 2015, Liu et al. 2015, Jugo et al. 2014, Coffrin et al. 2014, García et al. 2012, Martínez et al. 2011, Paralič et al. 2011, Li \& Huang 2008]. Dentre os 60 estudos excluídos nesta etapa, 41 o foram em função do CE1, 12 em função do CE4, 4 em função do CE3, e 3 em função do CE2.

A partir deste conjunto final de onze trabalhos, foi realizada a leitura completa dos artigos de forma a responder às questões de pesquisa deste estudo. A próxima seção descreve os resultados desta análise.

\section{Análise e Interpretação dos Resultados}

Durante a leitura dos onze artigos selecionados, foram analisados seus objetivos, fundamentação teórica, metodologias e resultados, e realizadas as etapas de "keywording", classificação e sumarização, conforme proposto por Petersen (2007). As próximas subseções apresentam os resultados obtidos, separados por cada uma das questões de pesquisa descritas na Subseção 3.1.

\subsection{Q1: Que aspectos dos processos de aprendizagem as pesquisas estão buscando analisar a partir dos dados armazenados nas plataformas de aprendizagem?}

Por meio desta questão de pesquisa, buscou-se identificar as abordagens adotadas pelos estudos para representar processos cognitivos através de visualizações de dados. A abordagem mais comum dentre os estudos analisados foi a visualização de trajetórias de aprendizagem realizadas, presente nos trabalhos de Gelan et al. (2018), Jugo et al. (2014), Coffrin et al. (2014) e Paralič et al. (2011). Uma "trajetória de aprendizagem realizada" representa, segundo Hannel et al. (2016), "o conjunto de atividades realizadas por um indivíduo com o objetivo de alcançar um determinado objetivo educacional". Nos trabalhos que focaram neste aspecto, os autores analisaram a sequência temporal de

\footnotetext{
1 Embora artigos em Português não tenham sido incluídos, boa parte dos melhores trabalhos desenvolvidos em países lusófonos estariam, em tese, contemplados, pois são publicados em Inglês, em conferências e periódicos internacionais.
} 
VII Congresso Brasileiro de Informática na Educação (CBIE 2018)

Anais do XXIX Simpósio Brasileiro de Informática na Educação (SBIE 2018)

atividades desempenhadas pelos discentes na plataforma virtual (individualmente ou de forma agregada) exclusivamente a partir dos logs ou combinando logs com fontes externas de dados.

Além das trajetórias de aprendizagem realizadas, outros focos de análise abordados foram: participação em fóruns de discussão [Hernández-García et al. 2015, Li \& Huang 2008], rotina de estudo individual vs em grupo [Zhang et al. 2018], tempo investido e frequência na realização de atividades propostas [Gómez-Aguilar et al. 2015], processos envolvidos na construção de um artefato digital [Liu et al. 2015, Martínez et al. 2011] e atividades realizadas fora do AVA da instituição [García et al. 2012].

Dentro das abordagens descritas acima, os autores investigaram diferentes processos cognitivos. A Figura 3 mostra a distribuição dos tipos de processos cognitivos analisados, evidenciando que os processos de interação e participação foram os mais comuns, estando presente em cinco trabalhos [Hernández-García et al. 2015, Gómez-Aguilar et al. 2015, Coffrin et al. 2014, García et al. 2012, Li \& Huang 2008]. Estes dois processos foram agrupados em uma única categoria pelo fato de muitas teorias da aprendizagem considerá-los sinônimos (por exemplo, em [Rogoff 2003] e [Lave \& Wenger 1991]), embora muitas vezes o termo interação seja utilizado com o sentido de "comunicação entre pares" e participação com o sentido de "participação em atividades".

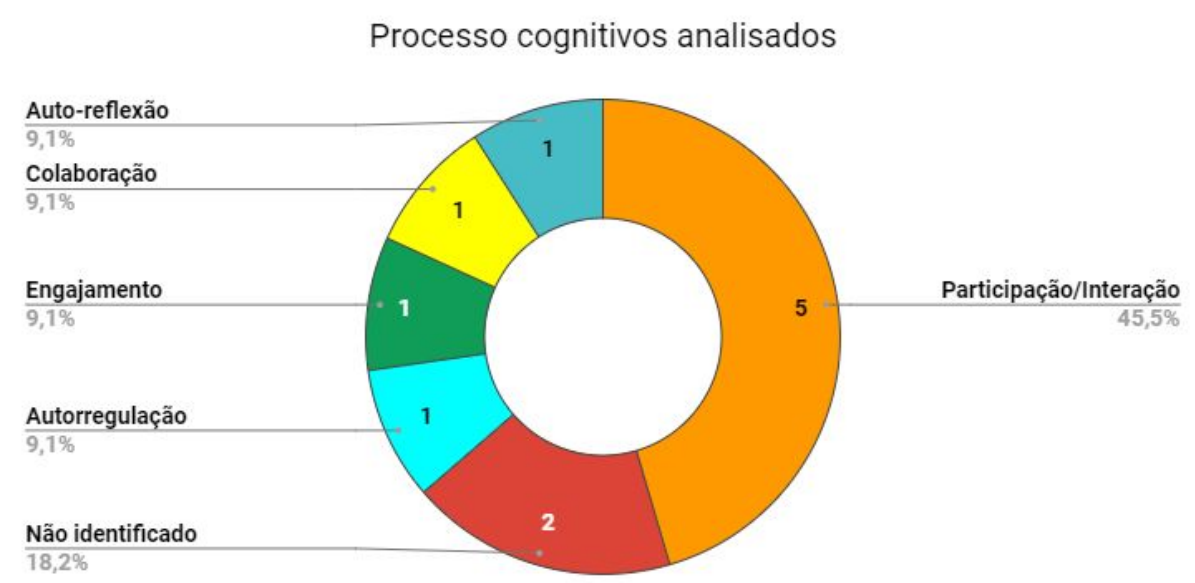

Figura 2. Distribuição dos tipos de processos analisados pelos estudos.

\subsection{Q2: Que técnicas de visualização têm sido utilizadas na concepção dos artefatos?}

A partir da pergunta desta questão de pesquisa, buscou-se analisar os tipos de visualização mais utilizados nos estudos, bem como a presença de recursos de interatividade nas visualizações propostas. A Tabela 1 mostra os tipos de visualização identificados e suas frequências. É possível observar que histogramas, grafos (usados para representar redes sociais), gráficos de linhas e diagramas de linha do tempo foram as metáforas mais utilizadas.

No que tange à sofisticação e adequabilidade das visualizações utilizadas, destacam-se os trabalhos de Gómez-Aguilar et al. (2015) e Paralič et al. (2011). No 
VII Congresso Brasileiro de Informática na Educação (CBIE 2018)

Anais do XXIX Simpósio Brasileiro de Informática na Educação (SBIE 2018)

primeiro, os autores propõem um novo tipo de visualização, Semantic Spiral Timeline (SST), que consiste num diagrama de linha do tempo em forma de "espiral deformada", com o intuito de representar sequência de eventos e evidenciar períodos de inatividade. Os autores implementaram este conceito numa ferramenta que oferece também recursos de interatividade como filtragem e agrupamento dos dados, além de vistas coordenadas. Já no trabalho de Paralič et al. (2011), os autores propõem e implementam uma linha do tempo também interativa, que permite aos estudantes, colaborativamente, buscar e visualizar padrões comportamentais a partir de logs de realização de atividades em um AVA.

Tabela 1. Tipos de visualização e frequências nos estudos analisados

\begin{tabular}{|l|r|}
\hline Tipos de visualização & Frequência \\
\hline Histograma & 3 \\
\hline Grafo & 3 \\
\hline Gráfico de linhas & 3 \\
\hline Linha do tempo & 3 \\
\hline Diagrama de estados & 2 \\
\hline Setores & 2 \\
\hline Radar & 2 \\
\hline Outros (Barras, Scatterplot, Starburst, CDF, Heatmap) & 5 \\
\hline
\end{tabular}

Embora os dois trabalhos mencionados apresentem propostas de visualizações interativas, este não é o cenário mais comum - apenas três dos onze estudos $(27,3 \%)$ propõem ferramentas com algum nível de interatividade, sendo o terceiro o estudo de García et al. 2012. Este cenário vai de encontro com a literatura de visualização de dados, que considera a interatividade essencial para que o usuário seja capaz de extrair informações relevantes de uma visualização [Muzner 2014; Yi, Kang \& Stasko 2007].

\subsection{Q3: As visualizações propostas foram submetidas a avaliação de atores envolvidos no processo educacional (alunos, professores, gestores) ou incorporadas à suas práticas?}

A terceira questão de pesquisa deste estudo busca verificar se houve validação das visualizações propostas com os atores interessados (alunos, professores, gestores, entre outros), e não apenas pesquisadores, como preconizado pela corrente do Design Centrado no Usuário [Norman \& Draper 1986]. Neste aspecto, a maioria dos estudos $(72,7 \%)$ não realizaram testes de usabilidade ou aceitação para confirmar o valor entregue pelas suas propostas.

Apenas os trabalhos de Liu et al. (2015), Martínez et al. (2011) e Paralič et al. (2011) submeteram suas propostas a testes com usuários. Paralič et al. (2011) realizaram testes com alunos e instrutores, e também compararam o uso da ferramenta digital com uma versão analógica (usando papel, canetas e adesivos para montar uma linha do tempo de eventos). Martínez et al. (2011) testaram o uso do dashboard desenvolvido a partir de um dispositivo móvel em que os instrutores poderiam acompanhar, em tempo real, indicadores de colaboração de estudantes desenvolvendo uma atividade em equipe numa mesa gráfica. Por fim, Liu et al. (2015) analisaram a confiabilidade das 
VII Congresso Brasileiro de Informática na Educação (CBIE 2018)

Anais do XXIX Simpósio Brasileiro de Informática na Educação (SBIE 2018)

visualizações quando comparadas com observação in situ e com a própria percepção dos estudantes sobre seus níveis de engajamento numa atividade de produção textual.

\subsection{Q4: Que teorias da aprendizagem têm sido usadas para guiar a investigação?}

Alguns pesquisadores apontam a importância de se utilizar teorias da aprendizagem como alicerce para a construção de sistemas de análise visual de dados educacionais efetivos [Vieira et al. 2018, Bogarín 2018]. No entanto, como mostrado na Figura 4, apenas dois trabalhos $(18,2 \%)$ utilizaram efetivamente alguma teoria da aprendizagem como alicerce para construção de suas visualizações: Liu et al. (2015) e Paralič et al. (2011). No primeiro, os autores basearam-se no conceito de "engajamento comportamental" de R. D. Jones [Jones 2009]. Jones define três tipos de engajamento (comportamental, emocional e cognitivo) e três dimensões associadas: intensidade, que exprime o nível de engajamento de cada estudante; consistência, denotando por quanto tempo os estudantes permanecem altamente engajados ao longo da aula; e profundidade, que mede o quanto a classe como um todo está engajada na atividade. No segundo, os autores utilizaram a noção de "trialogical approach to learning" [Paavola \& Hakkarainen 2009], que se coloca como alternativa às abordagens classificadas por Paavola e Hakkarainen como "monological" (cognitiva) e "dialogical" (situada).

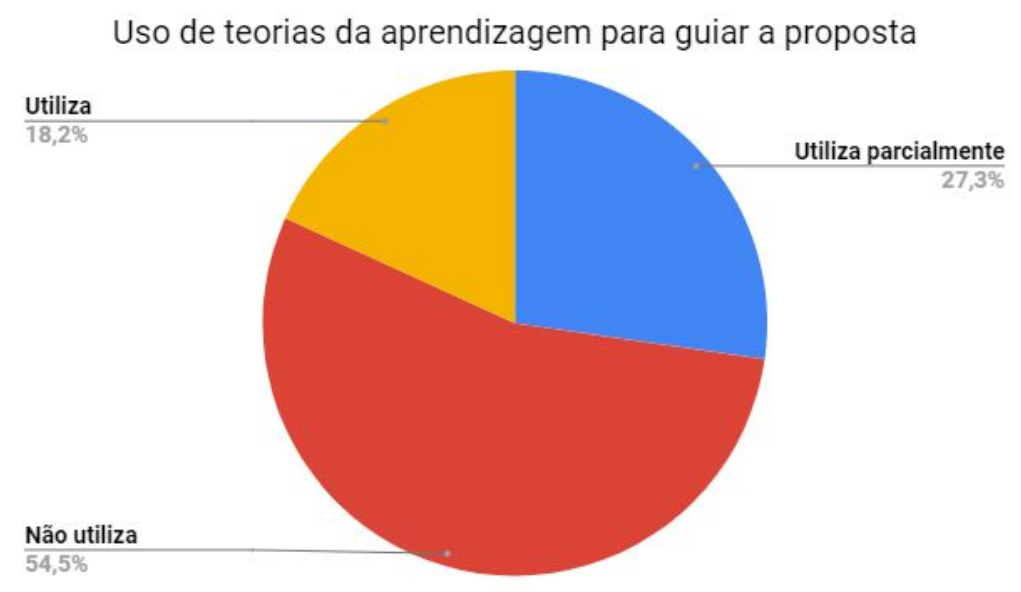

Figura 3. Utilização de teorias da aprendizagem nos estudos analisados

Outros três trabalhos $(27,3 \%)$ utilizaram parcialmente alguma teoria da aprendizagem em suas investigações, porém de forma marginal, e não como alicerce teórico para o estudo: Gelan et al. (2018), que cita a teoria da autorregulação da aprendizagem segundo o entendimento de Butler \& Winne (1995); e Gómez-Aguilar et al. (2015) e García et al. (2012), que citam o trabalho de Anderson \& Garrison (1998) sobre tipos de interação em AVAs.

\section{Considerações Finais}

A análise processual da aprendizagem em AVAs é apontada por alguns autores como um tema pouco explorado na literatura. Este estudo buscou investigar, através de um mapeamento sistemático, como os trabalhos na área de Visual Learning Analytics têm atacado este problema. A partir das questões de pesquisa adotadas, foram identificados os seguintes padrões e lacunas na literatura: i) a análise de "trajetórias de aprendizagem 
VII Congresso Brasileiro de Informática na Educação (CBIE 2018)

Anais do XXIX Simpósio Brasileiro de Informática na Educação (SBIE 2018)

realizadas" foi a abordagem mais comum para investigação dos processos de aprendizagem; ii) poucos trabalhos apresentaram propostas de visualizações com recursos de interatividade; iii) poucos trabalhos validaram suas propostas com os atores envolvidos no processo de ensino-aprendizagem; e iv) apenas dois trabalhos utilizaram efetivamente teorias da aprendizagem para alicerçar suas propostas.

Desse modo, os resultados deste estudo apontam para a necessidade de mais trabalhos na área de Visual Learning Analytics com propostas de visualizações sofisticadas, interativas, embasadas em teorias da aprendizagem e validadas empiricamente, capazes de explicitar os processos de aprendizagem em AVAs.

\section{Agradecimentos}

Raphael Dourado é bolsista de doutorado do CNPq, processo no 140973/2017-6. Alex Sandro Gomes é Pesquisador DTI Nível 2 CNPq nos processos 310.466/2012-1, 475634/2013-6 e 307202/2015-1.

\section{Referências}

ANDERSON, Terry; GARRISON, D. Randy. Learning in a networked world: New roles and responsibilities. In: Distance Learners in Higher Education: Institutional responses for quality outcomes. Madison, Wi.: Atwood. 1998.

ARKSEY, Hilary; O'MALLEY, Lisa. Scoping studies: towards a methodological framework. International journal of social research methodology, v. 8, n. 1, p. 19-32, 2005.

BOGARÍN, Alejandro; CEREZO, Rebeca; ROMERO, Cristóbal. A survey on educational process mining. Wiley Interdisciplinary Reviews: Data Mining and Knowledge Discovery, v. 8, n. 1, p. e1230, 2018.

BUTLER, Deborah L.; WINNE, Philip H. Feedback and self-regulated learning: A theoretical synthesis. Review of educational research, v. 65, n. 3, p. 245-281, 1995.

COFFRIN, Carleton et al. Visualizing patterns of student engagement and performance in MOOCs. In: Proceedings of LAK'14. ACM, 2014. p. 83-92.

GARCÍA, C.; Raquel M. et al. Peeking into the black box: visualising learning activities. International Journal of Technology Enhanced Learning, v. 4, n. 1-2, p. 99-120, 2012.

GELAN, Anouk et al. Affordances and limitations of learning analytics for computer-assisted language learning: a case study of the VITAL project. Computer Assisted Language Learning, v. 31, n. 3, p. 294-319, 2018.

GÓMEZ-AGUILAR, Diego Alonso et al. Tap into visual analysis of customization of grouping of activities in eLearning. Computers in Human Behavior, v. 47, p. 60-67, 2015.

HANNEL, K. (Org.) ; ZUNGUZE, M. C. (Org.) ; NUNES, F. B. (Org.) ; LIMA, J. V. (Org.). Trajetórias de Aprendizagem: teoria e prática. 1. ed. Create Space, 2016.

HERNÁNDEZ-GARCÍA, Ángel et al. Applying social learning analytics to message boards in online distance learning: A case study. Computers in Human Behavior, v. 47, p. 68-80, 2015.

JONES, R. D. Student Engagement: Teacher Handbook, Rexford:NY: International Center for Leadership in Education, 2009. 
VII Congresso Brasileiro de Informática na Educação (CBIE 2018)

Anais do XXIX Simpósio Brasileiro de Informática na Educação (SBIE 2018)

JUGO, Igor; KOVAČIĆ, Bozidar; SLAVUJ, Vanja. Using data mining for learning path recommendation and visualization in an intelligent tutoring system. In: 37th MIPRO. IEEE, 2014. p. 924-928.

LAVE, J.; WENGER, E. Situated Learning: Legitimate Peripheral Participation. Cambridge, UK: Cambridge University Press, 1991.

LI, Yanyan; HUANG, Ronghuai. Analyzing peer interactions in computer-supported collaborative learning: Model, method and tool. In: International Conference on Hybrid Learning and Education. Springer, Berlin, Heidelberg, 2008. p. 125-136.

LIU, Ming et al. Measuring and visualizing students' behavioral engagement in writing activities. IEEE Transactions on learning technologies, v. 8, n. 2, p. 215-224, 2015.

LOCKYER, Lori; HEATHCOTE, Elizabeth; DAWSON, Shane. Informing pedagogical action: Aligning learning analytics with learning design. American Behavioral Scientist, v. 57, n. 10, p. 1439-1459, 2013.

MARTÍNEZ, Roberto et al. Who did what? Who said that?: Collaid: an environment for capturing traces of collaborative learning at the tabletop. In: Proc. of the ACM International Conference on Interactive Tabletops and Surfaces. 2011. p. 172-181.

MELLO, S. A (2004). A Escola de Vygotsky. In: CARRARA, K. (Org.) Introdução à psicologia da educação: seis abordagens. São Paulo: Avercamp, p. 135-155.

MUNZNER, T. Visualization analysis and design. [S.1.]: CRC press, 2014.

NORMAN, D. and DRAPER, S. User Centered System Design; New Perspectives on Human-Computer Interaction. L. Erlbaum Assoc. Inc., Hillsdale, NJ, USA:1986.

PAAVOLA, S. \& HAKKARAINEN, K.. From Meaning Making to Joint Construction of Knowledge Practices and Artefacts - A Trialogical Approach to CSCL. In C. O'Malley, D. Suthers, P. Reimann, \& A. Dimitracopoulou (Eds.), Computer Supported Collaborative Learning Practices: CSCL2009 Proceedings, 83-92, ISBN 978-1-4092-8598-4, 2009.

PARALIČ, Ján et al. Mirroring of knowledge practices based on user-defined patterns. Journal of Universal Computer Science, v. 17, n. 10, p. 1474-1491, 2011.

PETERSEN, Kai et al. Systematic Mapping Studies in Software Engineering. In: EASE. 2008. p. 68-77.

ROGOFF, Barbara. The cultural nature of human development. Oxford Univ. Press, 2003.

SIEMENS, George; GASEVIC, Dragan. Guest editorial-Learning and knowledge analytics. Educational Technology \& Society, v. 15, n. 3, p. 1-2, 2012.

VIEIRA, Camilo; PARSONS, Paul; BYRD, Vetria. Visual learning analytics of educational data: A systematic literature review and research agenda. Computers \& Education, v. 122, p. 119-135, 2018.

YI, J. S.; KANG, Y. \& STASKO, J. Toward a deeper understanding of the role of interaction in information visualization. IEEE Transactions on Visualization and Computer Graphics, v.13, n.6, p.1224-1231, 2007.

ZHANG, Jia-Hua et al. What Learning Analytics Tells Us: Group Behavior Analysis and Individual Learning Diagnosis based on Long-Term and Large-Scale Data. Journal of Educational Technology \& Society, v. 21, n. 2, p. 245-258, 2018. 